\title{
Three-dimensional, multispecies, high spatial resolution MHD studies of the solar wind interaction with Mars
}

\author{
Yingjuan Ma, Andrew F. Nagy, Igor V. Sokolov, and Kenneth C. Hansen \\ Space Physics Research Laboratory, Department of Atmospheric, Oceanic, and Space Sciences, University of Michigan, \\ Ann Arbor, Michigan, USA \\ Received 22 December 2003; revised 5 May 2004; accepted 1 June 2004; published 24 July 2004.
}

[1] We present the results of model calculations, using our new, four-species, spherical MHD model. Our results are compared with the relevant and limited available data. The resulting comparisons help us to increase our understanding of the interaction processes between the solar wind and the Martian atmosphere/ionosphere. This new model with a spherical grid structure allowed us to use small $(\sim 10 \mathrm{~km})$ radial grid spacing in the ionospheric region. We found that the calculated bow shock positions agree reasonably well with the observed values. The calculated results vary with interplanetary magnetic field orientation, solar cycle conditions, and subsolar location. We found that our calculated ion densities, with parameters corresponding to solar cycle minimum conditions, reproduced the Viking 1 observed ion densities well. The calculated solar cycle maximum densities, above $\sim 140 \mathrm{~km}$, are also consistent with the appropriate Mars Global Surveyor radio occultation electron densities. Both the calculated solar cycle maximum and solar cycle minimum total transterminator and escape fluxes are significantly smaller than our previously published values. This decrease is due to the improved temperature values used for the recombination rates in this new model, which in turn results in lower ion densities and lower fluxes. INDEX TERMS: 2780 Magnetospheric Physics: Solar wind interactions with unmagnetized bodies; 6026 Planetology: Comets and Small Bodies: Ionospherescomposition and chemistry; 6028 Planetology: Comets and Small Bodies: Ionospheres-structure and dynamics; 2728 Magnetospheric Physics: Magnetosheath; KEYWORDS: Mars, MHD, bow shock, ionosphere, solar wind interaction

Citation: Ma, Y., A. F. Nagy, I. V. Sokolov, and K. C. Hansen (2004), Three-dimensional, multispecies, high spatial resolution MHD studies of the solar wind interaction with Mars, J. Geophys. Res., 109, A07211, doi:10.1029/2003JA010367.

\section{Introduction}

[2] The purpose of this paper is to present the results of model calculations using our new, four-species, high radial resolution MHD model and to compare these results with the relevant and limited data available. Such comparisons are a powerful tool in providing help to elucidate the physical processes controlling the interaction of the solar wind with Mars.

[3] Studies of the interaction of the solar wind with Mars and Venus, using MHD approaches, date back more than a decade [e.g., Steinolfson and Cable, 1993; Sauer et al., 1994; DeZeeuw et al., 1996; Tanaka and Murawski, 1997; Bauske et al., 1998; Kallio et al., 1998; Sauer et al., 1998; Liu et al., 1999; Ma et al., 2002; Harnett and Winglee, 2003]. Over the years our group has been working on this problem and adopted the "incremental improvement" approach. We started out with singlespecies, axisymmetric assumptions, and in our last paper we presented results from a three-species, three-dimensional (3-D) model of the solar wind interaction with Mars, including the effects of the crustal magnetic field and mass loading [Ma et al., 2002]. That model used adaptive, Cartesian coordinates, but still the minimum grid size was limited to about $50 \mathrm{~km}$. The purpose of this paper is to present the results from our latest model calculations. In this model the grid structure was changed to a spherical one, which allows an altitude resolution of about $10 \mathrm{~km}$ in the ionosphere, which is comparable to the neutral gas and thus the ionization scale heights. This much-improved altitude resolution means that within the limitations of the MHD approximations, the model results provide meaningful ionospheric parameters and a better resolution in the rest of the interaction region.

\section{Model Description}

[4] We use the ideal MHD equations in our model. More specifically, the results to be presented here are based on our latest model, which uses four continuity, one momentum, one magnetic induction, and one energy equation. The specific, normalized equations solved are

$$
\frac{\partial \mathbf{W}}{\partial \mathrm{t}}+\{\nabla \cdot \mathbf{F}\}^{\mathrm{T}}=\mathbf{Q}
$$


Table 1. List of Chemical Reactions and Rates Considered in the Model

\begin{tabular}{lll}
\hline \multicolumn{1}{c}{ Reaction } & \multicolumn{1}{c}{ Rate Coefficient } & \multicolumn{1}{c}{ References } \\
\hline $\mathrm{CO}_{2}+\mathrm{h} \nu \rightarrow \mathrm{CO}_{2}^{+}+\mathrm{e}$ & $7.30 \times 10^{-7} \mathrm{~s}^{-1}$ (solar max) & Schunk and Nagy [2000] \\
& $2.47 \times 10^{-7} \mathrm{~s}^{-1}$ (solar min) & \\
$\mathrm{O}+\mathrm{h} \nu \rightarrow \mathrm{O}^{+}+\mathrm{e}$ & $2.73 \times 10^{-7} \mathrm{~s}^{-1}$ (solar max) & Schunk and Nagy [2000] \\
& $8.89 \times 10^{-8} \mathrm{~s}^{-1}($ solar min) & \\
$\mathrm{H}+\mathrm{h} \nu \rightarrow \mathrm{H}^{+}+\mathrm{e}$ & $8.59 \times 10^{-8} \mathrm{~s}^{-1}($ solar max) & J. Fox (private communication, 2004) \\
& $5.58 \times 10^{-8} \mathrm{~s}^{-1}($ solar min) & \\
$\mathrm{CO}_{2}^{+}+\mathrm{O} \rightarrow \mathrm{O}_{2}^{+}+\mathrm{CO}$ & $1.64 \times 10^{-10} \mathrm{~cm}^{-3} \mathrm{~s}^{-1}$ & Schunk and Nagy [2000] \\
$\mathrm{CO}_{2}^{+}+\mathrm{O} \rightarrow \mathrm{O}^{+}+\mathrm{CO} 2$ & $9.60 \times 10^{-11} \mathrm{~cm}^{-3} \mathrm{~s}^{-1}$ & Schunk and Nagy [2000] \\
$\mathrm{O}^{+}+\mathrm{CO} \rightarrow \mathrm{O}_{2}^{+}+\mathrm{CO}$ & $1.1 \times 10^{-9}(800 / \mathrm{Ti})^{0.39} \mathrm{~cm}^{-3} \mathrm{~s}^{-1}$ & Fox and Sung [2001] \\
$\mathrm{O}^{+}+\mathrm{H} \rightarrow \mathrm{H}^{+}+\mathrm{O}$ & $6.4 \times 10^{-10} \mathrm{~cm}^{-3} \mathrm{~s}^{-1}$ & Schunk and Nagy [2000] \\
$\mathrm{H}^{+}+\mathrm{O} \rightarrow \mathrm{O}^{+}+\mathrm{H}$ & $5.08 \times 10^{-10} \mathrm{~cm}^{-3} \mathrm{~s}^{-1}$ & Fox and Sung [2001] \\
$\mathrm{O}_{2}^{+}+\mathrm{e} \rightarrow \mathrm{O}+\mathrm{O}$ & $7.38 \times 10^{-8}(1200 / \mathrm{Te})^{0.56} \mathrm{~cm}^{-3} \mathrm{~s}^{-1}$ & Schunk and Nagy [2000] \\
$\mathrm{CO}_{2}^{+}+\mathrm{e} \rightarrow \mathrm{CO}+\mathrm{O}$ & $3.10 \times 10^{-7}(300 / \mathrm{Te})^{0.5} \mathrm{~cm}^{-3} \mathrm{~s}^{-1}$ & Schunk and Nagy [2000]
\end{tabular}

The state vector $\mathrm{W}$ and flux tensor $\mathrm{F}$ in equation (1) are

$$
\begin{gathered}
\mathbf{W}=\left(\begin{array}{c}
\rho_{\mathrm{i}} \\
\left(\sum_{\mathrm{i}=1}^{4} \rho_{\mathrm{i}}\right) \mathbf{u} \\
\mathbf{B} \\
\varepsilon
\end{array}\right)\left(\begin{array}{c}
\left(\sum_{\mathrm{i}=1}^{4} \rho_{\mathrm{i}}\right) \mathbf{u} \mathbf{u}+\left(\mathrm{p}+\frac{1}{2} \mathrm{~B}^{2}\right) \mathbf{I}-\mathbf{B B} \\
\mathbf{u B}-\mathbf{B} \mathbf{u} \\
\mathbf{u}\left(\varepsilon+\mathrm{p}+\frac{1}{2} \mathbf{B}^{2}\right)-(\mathbf{B} \cdot \mathbf{u}) \mathbf{B}
\end{array}\right),
\end{gathered}
$$

where $\varepsilon$ is the total energy density, defined as

$$
\varepsilon=\frac{1}{2}\left(\sum_{i=1}^{4} \rho_{i}\right) u^{2}+\frac{1}{\gamma-1} p+\frac{B^{2}}{2} .
$$

The source term in equation (1) is

$$
Q=\left(\begin{array}{c}
\left(\sum_{i=1}^{4} \rho_{\mathrm{i}}\right) \mathbf{g}-\left(\sum_{\mathrm{i}=1}^{4} \rho_{\mathrm{i}}\right) v \mathbf{u}-\mathbf{u}\left(\sum_{\mathrm{i}=1}^{4} \mathrm{~L}_{\mathrm{i}}\right) \\
0 \\
\left(\sum_{\mathrm{i}=1}^{4} \rho_{\mathrm{i}}\right) \mathbf{u} \cdot \mathbf{g}-\frac{1}{2} \mathrm{u}^{2}\left(\sum_{\mathrm{i}=1}^{4} \mathrm{~L}_{\mathrm{i}}\right)-\left(\sum_{\mathrm{i}=1}^{4} \rho_{\mathrm{i}}\right) \nu \mathrm{u}^{2}-\frac{\mathrm{p}}{\gamma-1}\left(\sum_{\mathrm{i}=1}^{4} \frac{\mathrm{L}_{\mathrm{i}}}{\mathrm{m}_{\mathrm{i}}} / \sum_{\mathrm{i}=1}^{4} \frac{\rho_{\mathrm{i}}}{\mathrm{m}_{\mathrm{i}}}\right) \\
+\frac{\mathrm{kT}}{\gamma-1}\left(\sum_{\mathrm{i}=1}^{4} \frac{\mathrm{S}_{\mathrm{i}}}{\mathrm{m}_{\mathrm{i}}}\right)-4 \times 10^{-11} \sum_{i=1}^{3} \mathrm{n}_{\mathrm{i}} \sum_{\mathrm{i}=1}^{4} \frac{\rho_{\mathrm{i}}}{\mathrm{m}_{\mathrm{i}}} \exp \left[10\left(\mathrm{~T}_{\mathrm{p}}-6000\right) / \mathrm{T}_{\mathrm{p}}\right]
\end{array}\right),
$$

where $\rho_{1}, \rho_{2}, \rho_{3}$, and $\rho_{4}$ are the $\mathrm{H}^{+}, \mathrm{O}_{2}^{+}, \mathrm{O}^{+}$, and $\mathrm{CO}_{2}^{+}$mass densities, respectively, $\mathrm{S}_{1}, \mathrm{~S}_{2}, \mathrm{~S}_{3}, \mathrm{~S}_{4}$ and $\mathrm{L}_{1}, \mathrm{~L}_{2}, \mathrm{~L}_{3}, \mathrm{~L}_{4}$ are the $\mathrm{H}^{+}, \mathrm{O}_{2}^{+}, \mathrm{O}^{+}$, and $\mathrm{CO}_{2}^{+}$mass production and loss rates, respectively, $\mathrm{p}$ is the total thermal pressure of the plasma, $\mathbf{u}$ is the velocity of the plasma, $\nu$ is the ion neutral collision frequency (taken to be $4 \times 10^{-10}\{[\mathrm{O}]+[\mathrm{CO} 2]+$ $\left.[\mathrm{H}]\} \mathrm{s}^{-1}\right), \mathrm{T}_{\mathrm{o}}$ is the temperature of the newly produced ions, which is assumed to be the same as the temperature of local neutral atmosphere, $\gamma$ is the ratio of specific heats (and taken to be 5/3), and the other symbols have their usual definition. The last term in equation (5) is a "physics-based ad hoc" term and it acts to keep the temperatures close to the observed values in the ionosphere. An analogous cooling term was used by Tanaka and Murawski [1997] in their MHD model to for Venus, in order to keep the ionospheric temperatures "reasonable." In equation (5), $\mathrm{T}_{\mathrm{p}}$ is plasma temperature, and $\mathrm{n}_{1}, \mathrm{n}_{2}, \mathrm{n}_{3}$ are the number densities of neutral component, $\mathrm{CO}_{2}, \mathrm{O}$, and $\mathrm{H}$, respectively.

[5] The system of equations solved considers only a single momentum equation and thus calculates only one velocity. This is certainly a simplifying approximation, but we believe it still leads to meaningful results because the transition from proton-dominated to oxygen-dominated regions is rapid, and in the ionosphere where the $\mathrm{O}^{+}$and $\mathrm{O}_{2}^{+}$densities are comparable, the ion velocities are expected to be about the same.

[6] The chemical reactions that we used in this four-ion model are listed in Table 1. A number of reaction rates are temperature-dependent. In order to evaluate these rates, we assumed that the electron and ion temperatures are approximately equal and given by $\mathrm{T}_{\mathrm{p}} / 2$.

[7] We approximated the optical depth effect by including a cosine factor for the different solar zenith angles and by assuming average absorption coefficients of $2.6 \times$ $10^{-17}$ and $1.5 \times 10^{-17} \mathrm{~cm}^{2}$ for $\mathrm{CO}_{2}$ and $\mathrm{O}$, respectively [Schunk and Nagy, 2000]. On the nightside, the solar flux was assumed to be $1.0 \times 10^{-5}$ times the unattenuated solar radiation in order to avoid zeros. The neutral densities used in the calculations for solar maximum and solar minimum conditions are shown in Figure 1 and Figure 2, respectively. For solar minimum condition the $\mathrm{CO}_{2}$ density values were based on the Viking neutral mass spectrometer data [Nier and McElroy, 1977] and the thermal oxygen values were based on the retarding potential analyzer (RPA) observations from Viking [Hanson et al., 1977]. The hydrogen densities used were those from Fox [2003] and the hot oxygen densities came from the calculations of Kim et al. [1998]. The $\mathrm{CO}_{2}$ and thermal oxygen densities, for solar cycle maximum, were from the model calculations of Bougher et al. [2000]; these model results were constrained by the Mariner 6-7 observations. The neutral gas temperatures were taken to be consistent with the Mariner 6-7 observations for solar maximum and the 


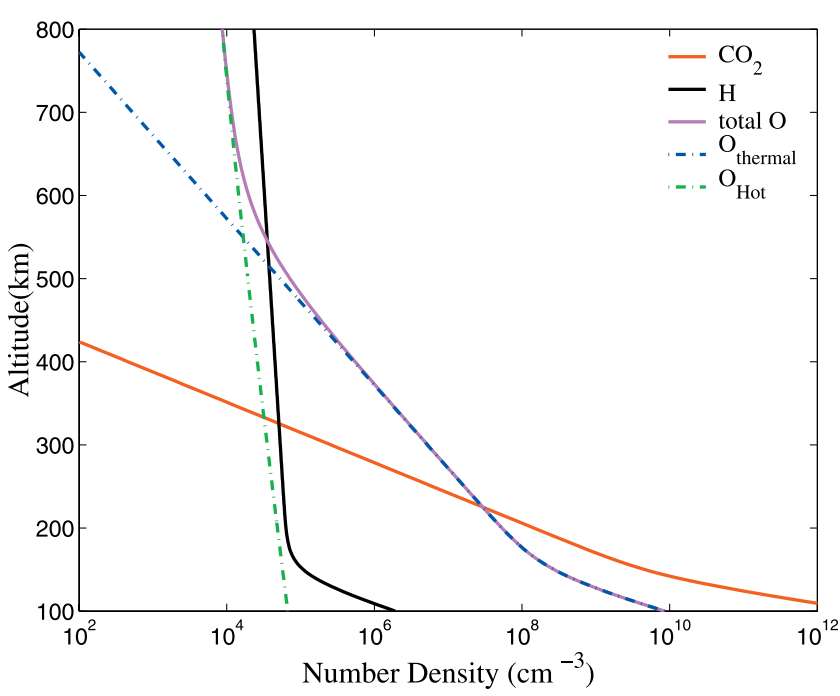

Figure 1. Density profiles of the neutral species adopted for solar cycle maximum conditions.

Viking results [Nier and McElroy, 1977] for solar minimum conditions.

[8] We used the 60 degree harmonic expansion for the crustal magnetic field, developed by Arkani-Hamed [2001], to describe the observed fields at Mars [Acuna et al., 1998]. We also ran a case corresponding to no crustal fields, for solar minimum conditions, to evaluate the effect of the crustal sources.

\section{Numerical Method}

[9] A modified version of the BATS-R-US (Block Adaptive Tree Solar Wind Roe-Type Upwind Scheme) has been used to solve the four-species MHD equations described above. The BATS-R-US solution method is a highly scalable, massively parallel, block-adaptive mesh refinement (block-AMR) algorithm developed for space physics applications that makes use of recent algorithmic advances in high-resolution upwind schemes. This family of numerical schemes can be applied for solving the hyperbolic systems of conservation laws (among them being the MHD system of equations), ensuring the high quality of the results, on one hand, and stability as well as the absence of spurious oscillations near sharp gradients, like shock waves, on the other hand. Details of the approach have been described elsewhere [Powell et al., 1999].

[10] Since we last presented results from a multispecies MHD model [Ma et al., 2002], our model underwent a number of improvements. The major change involved switching from a Cartesian to a spherical grid system in order to obtain much better altitude resolution, especially in the ionospheric regions (note we did not go to a spherical coordinate system but used a spherical grid structure). The grid is uniformly spaced, throughout each block, with respect to the natural logarithm of the radial distance, $r$, and the other two spherical coordinates $\Theta$ and $\Phi$. Each block contains $8 \times 4 \times 4$ cells. The cells were used as control volumes, confined by the planar faces, each of the faces being the middle perpendicular to the section connecting the adjacent grid meshes. It is easy to show that the control volumes were shaped as sections of pyramids and all the geometric characteristics of the control volumes, such as the volume $V_{i}$ and face area vectors $\mathbf{S}_{i j}$, can be calculated using elementary geometric considerations. The control volumes are constructed such that all faces between them are planar, the vector $\mathbf{S}_{i j}$ magnitude is nothing but the planar face area, and $\mathbf{S}_{i j}$ is directed from the $i$ th grid mesh to the neighboring $j$ th mesh. The integral formulation of equation (1), being applied to a control volume $V_{i}$, is

$$
\frac{\partial \mathbf{W}_{\mathrm{i}}}{\partial \mathrm{t}}+\frac{\sum_{\mathrm{j}}\left(\mathbf{S}_{\mathrm{ij}} \cdot \mathbf{F}_{\mathrm{ij}}\right)}{\mathrm{V}_{\mathrm{i}}}=\mathbf{Q}_{\mathrm{i}},
$$

where the sum is over the faces of the given control volume. Equation (6) allows the hydrodynamical flux through an arbitrarily oriented face to be obtained:

$$
(\mathbf{S} \cdot \mathbf{F})=\left(\begin{array}{c}
\rho_{\mathrm{i}}(\mathbf{S} \cdot \mathbf{u}) \\
\left(\sum_{\mathrm{i}=1}^{4} \rho_{\mathrm{i}}\right)(\mathbf{S} \cdot \mathbf{u}) \mathbf{u}+\left(\mathrm{p}+\frac{1}{2} \mathrm{~B}^{2}\right) \mathbf{S}-(\mathbf{S} \cdot \mathbf{B}) \mathbf{B} \\
(\mathbf{S} \cdot \mathbf{u}) \mathbf{B}-(\mathbf{S} \cdot \mathbf{B}) \mathbf{u} \\
(\mathbf{S} \cdot \mathbf{u})\left(\varepsilon+\mathrm{p}+\frac{1}{2} \mathrm{~B}^{2}\right)-(\mathbf{B} \cdot \mathbf{u})(\mathbf{S} \cdot \mathbf{B})
\end{array}\right) .
$$

[11] Both equations (1) and (6) thus have the same vector structure because the dot product of two vectors (e.g., $\mathbf{S} \cdot \mathbf{u}$ or $\nabla \cdot \mathbf{u})$ is a scalar. The resulting system of equations and all the physical vectors $\mathbf{S}, \mathbf{u}, \mathbf{B}$ can be thus taken in an arbitrarily chosen Cartesian frame of reference. Logarithmic spherical (curvilinear) coordinates are used only to define the grid mesh positions, and thus the solver did not need to be changed.

[12] The $x$ axis of our coordinate system points from Mars toward the Sun, $z$ axis is perpendicular to the $x$ axis and parallel to the projection of the North Pole on a plane

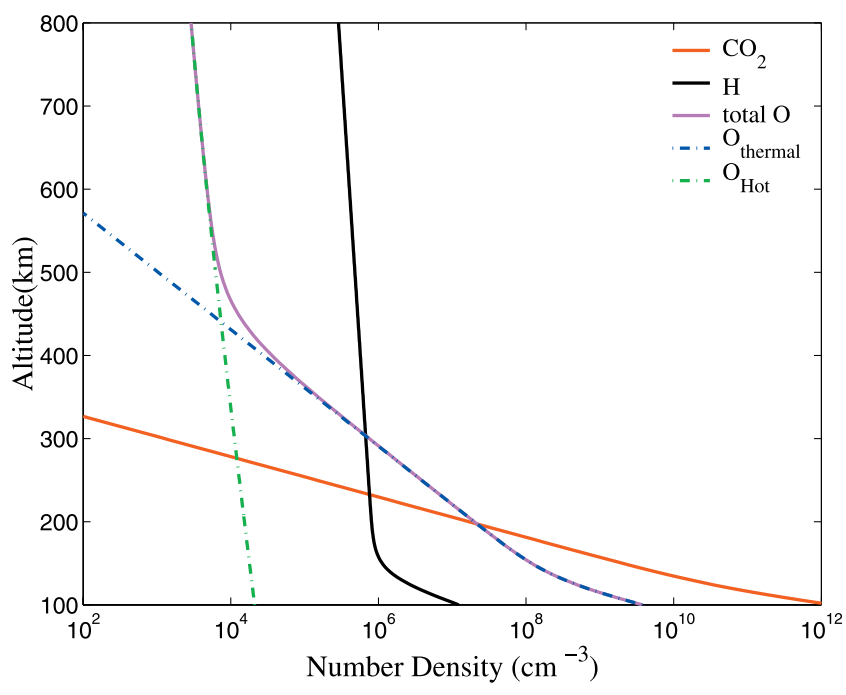

Figure 2. Density profiles of the neutral species adopted for solar cycle minimum conditions. 
Table 2. Related Parameters of Selected Cases and the Calculated Subsolar Bow Shock Location ${ }^{\mathrm{a}}$

\begin{tabular}{|c|c|c|c|c|c|c|c|}
\hline & Solar Cycle & $\begin{array}{l}\text { Crustal } \\
\text { Sources }\end{array}$ & $\begin{array}{l}\text { Interplanetary } \\
\text { Magnetic Field }\end{array}$ & $\begin{array}{l}\text { Subsolar } \\
\text { Position }\end{array}$ & $\begin{array}{c}\text { Subsolar Bow } \\
\text { Shock, } \mathrm{R}_{\mathrm{M}}\end{array}$ & $\begin{array}{c}\text { Averaged } \\
\text { Terminator Bow } \\
\text { Shock, } \mathrm{R}_{\mathrm{M}}\end{array}$ & $\begin{array}{c}\text { Subsolar } \\
\text { Magnetic Pileup } \\
\text { Boundary, } \mathrm{R}_{\mathrm{M}}\end{array}$ \\
\hline Case 1 & Maximum & Included & 3 nT Parker spiral & $180^{\circ} \mathrm{W} 0^{\circ} \mathrm{N}$ & 1.58 & 2.68 & 1.24 \\
\hline Case 2 & Maximum & Included & $\mathrm{B}_{\mathrm{y}}=3 \mathrm{nT}$ & $180^{\circ} \mathrm{W} 0^{\circ} \mathrm{N}$ & 1.59 & 2.73 & 1.24 \\
\hline Case 3 & Minimum & Included & 3 nT Parker spiral & $180^{\circ} \mathrm{W} 0^{\circ} \mathrm{N}$ & 1.54 & 2.58 & 1.22 \\
\hline Case 4 & Minimum & Included & 3 nT Parker spiral & $99.4^{\circ} \mathrm{W} 25.3^{\circ} \mathrm{N}$ & 1.43 & 2.44 & 1.13 \\
\hline Case 5 & Minimum & Not included & 3 nT Parker spiral & N/A & 1.39 & 2.33 & 1.11 \\
\hline
\end{tabular}

${ }^{\mathrm{a}}$ Observed subsolar bow shock location: (1.64 \pm 0.08$) \mathrm{R}_{\mathrm{M}}$ [Vignes et al., 2000], terminator bow shock location: (2.62 \pm 0.33$) \mathrm{R}_{\mathrm{M}}$ [Vignes et al., 2002], and subsolar magnetic pileup boundary location: (1.29 \pm 0.04$) \mathrm{R}_{\mathrm{M}}$ [Vignes et al., 2000].

perpendicular to the $x$ axis, and $y$ axis completes the righthand system. A computational domain which is defined by $-24 \mathrm{R}_{\mathrm{M}} \leq \mathrm{X} \leq 8 \mathrm{R}_{\mathrm{M}},-16 \mathrm{R}_{\mathrm{M}} \leq \mathrm{Y}, \mathrm{Z} \leq 16 \mathrm{R}_{\mathrm{M}}$, where $\mathrm{R}_{\mathrm{M}}=3396 \mathrm{~km}$ is the radius of Mars, was used in the calculations and the inner boundary was taken to be $100 \mathrm{~km}$ above the Martian surface. The nonuniform, spherical grid structure allowed a radial resolution that varied from $10 \mathrm{~km}$ at the lower boundary to $630 \mathrm{~km}$ at the outer boundary. The resolution with respect to the natural logarithm of the radial distance increased once, by a factor of two, at about $930 \mathrm{~km}$ altitude. At the same place, the angular resolution also increased from $1.875^{\circ}$ to $3.75^{\circ}$. The $\mathrm{O}_{2}^{+}, \mathrm{O}^{+}$, and $\mathrm{CO}_{2}^{+}$ densities at the inner boundary were taken to be the photochemical equilibrium value. A reflective boundary was used for $\mathbf{u}$; this boundary condition for $\mathbf{u}$ results in near zero velocities at the inner boundary, as expected. The plasma temperature (sum of the electron and ion temperatures) at the inner boundary was assumed to be two times the corresponding neutral temperature and the pressure was set accordingly. The upstream solar wind ion and electron temperatures were set to be $5 \times 10^{4}$ and $3 \times 10^{5}{ }^{\circ} \mathrm{K}$, respectively. The interplanetary magnetic field (IMF) was assumed to be a Parker spiral in the X-Y plane with an angle of 56 degrees and a magnitude of 3 nT (except for Case 2, where the IMF has only a $\mathrm{B}_{\mathrm{y}}$ component). The solar wind velocity and density were selected to be $400 \mathrm{~km} \mathrm{~s}^{-1}$ and $4 \mathrm{~cm}^{-3}$, respectively, for all the simulated cases. Detailed parameters for each run are listed in Table 2. For most of the cases we chose the subsolar location at 180 west longitude and 0 north latitude, while in Case 4 the subsolar location was taken to be at 99.4 west longitude and 25.3 north latitude, in order to closely approximate the Viking conditions.

\section{Simulation Results and Discussion}

[13] The calculated magnetic field and velocity results for Case 1 in the $\mathrm{X}-\mathrm{Y}$ and $\mathrm{X}-\mathrm{Z}$ planes are shown in Figures 3 and 4 , respectively. The white lines in the magnetic field plots show the field lines, and the white arrows in the velocity plot indicate the direction but not the magnitude of the velocity vectors. The general nature of these results is, as expected, very similar to the results obtained previously by our Cartesian model; the main differences are in the details obtained in the ionospheric regions. The flow pattern is upward and toward the terminator on the dayside, while on the nightside the flow is partially downward, helping to maintain the nightside ionosphere, as well as outward through the tail, contributing to the escape flux. The bow shock is clearly visible in both the velocity and magnetic
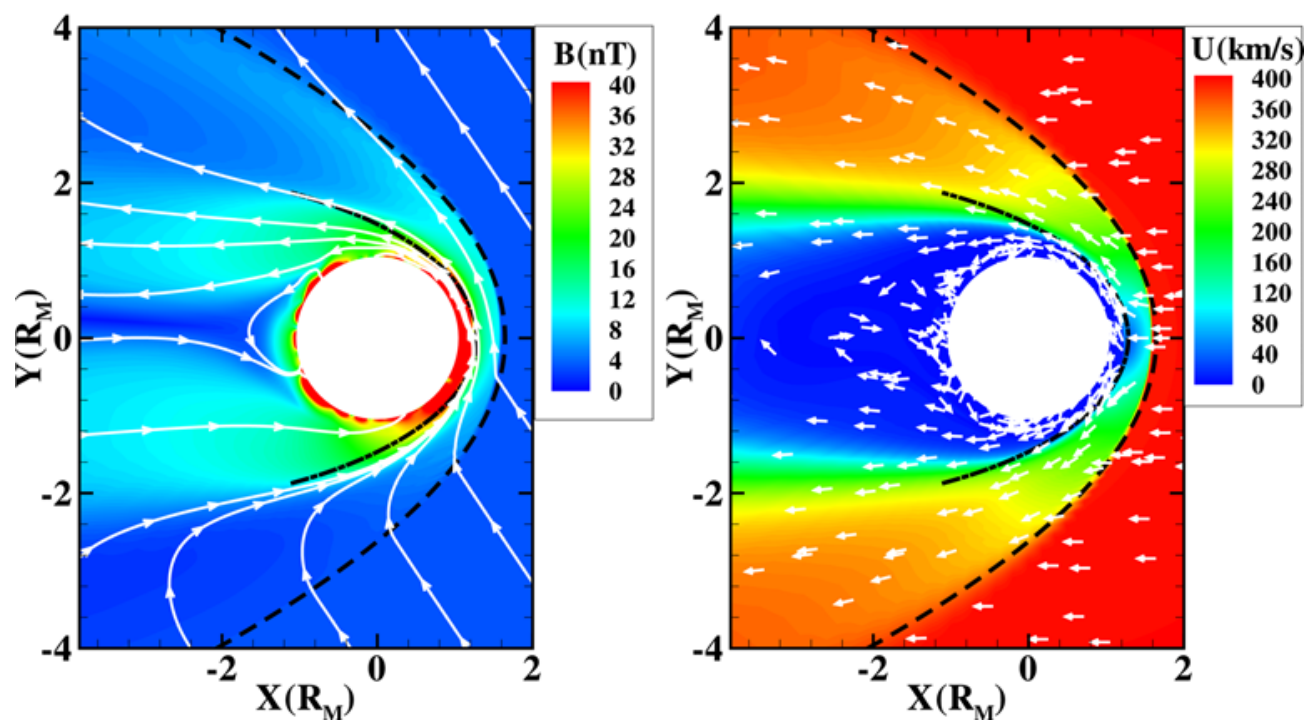

Figure 3. The calculated magnetic field and velocity in the equatorial plane for case 1 . The color plots show the magnitudes; the white lines marked with arrows indicate the vector direction of the magnetic field and the arrows show the direction (not the magnitude) of the velocity. The dashed line represents the mean bow shock and the dash-dot line is the mean MPB locations from Vignes et al. [2000]. 

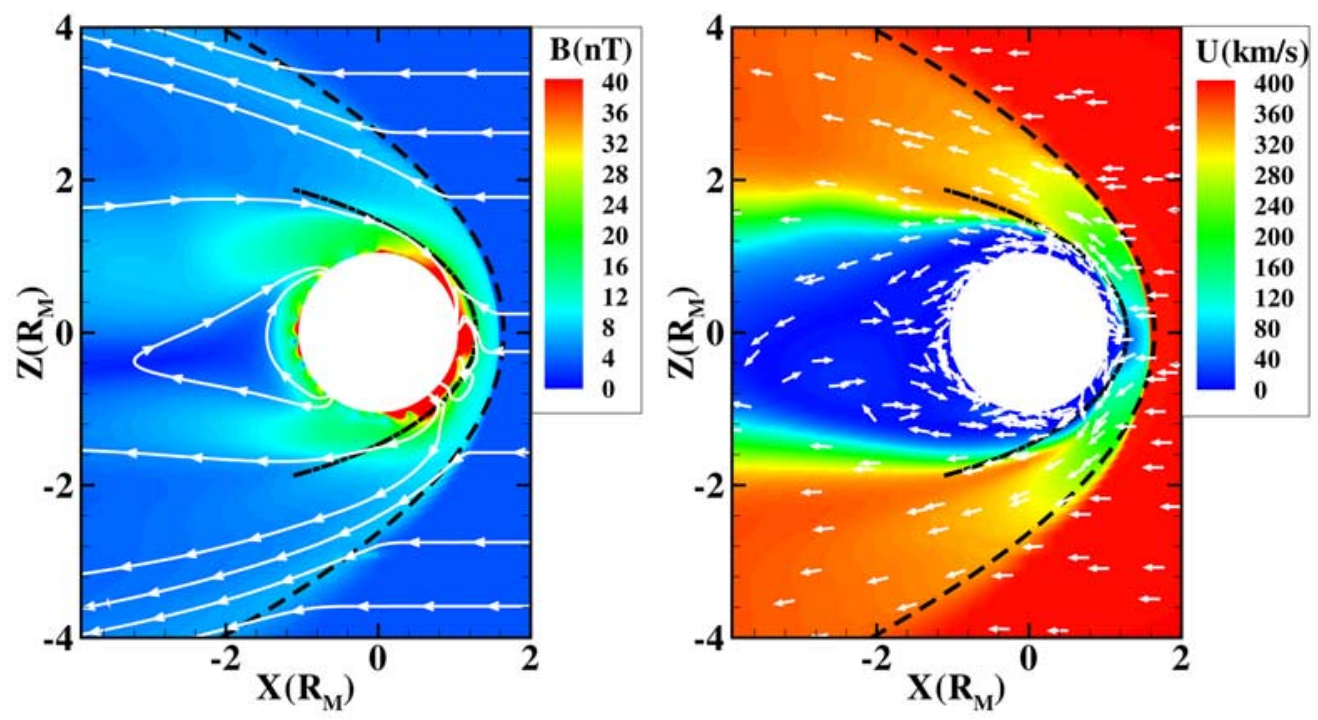

Figure 4. The calculated magnetic field and velocity in the meridianal plane for case 1 . The color plots show the magnitudes; the white lines marked with arrows indicate the vector direction of the magnetic field and the arrows show the direction (not the magnitude) of the velocity. The dashed line represents the mean bow shock and the dash-dot line is the mean MPB locations from Vignes et al. [2000].

field results. The calculated positions agree quite well with the average Mars Global Surveyor (MGS) results (shown by a dashed line) [Vignes et al., 2000]. The results presented here clearly show the draping of the interplanetary magnetic field, a magnetic pileup region (see also Figure 4), and the presence of minimagnetospheres. These features are wellknown characteristics of the plasma environment of Mars [cf. Nagy et al., 2004]. In the contour plot of the magnetic field in Figure 3 the shock position on the dawnside is closer than that at the duskside. This is consistent with MGS observations [Vignes et al., 2002], which showed that the quasi-parallel shocks are closer to the planet than the quasiperpendicular shocks. At the dawnside, quasi-parallel shocks dominate, while at the duskside quasi-perpendicular shocks dominate. The subsolar and averaged terminator bow shock locations for all the cases are given in Table 2 . In the current case the solar wind pressure is $1.06 \mathrm{nPa}$ and the corresponding subsolar bow shock is located at $1.58 \mathrm{R}_{\mathrm{M}}$. Change of the IMF orientation from the Parker spiral to purely y direction (Case 2) only results in a slight outward movement of the subsolar bow shock location and about $2 \%$ difference in the average terminator bow shock locations. Vignes et al. [2002] found that the mean bow shock position is independent of the solar cycle. Our results show that at solar cycle minimum conditions (Case 3 ) the bow shock moved inward toward the planet by $\sim 3-4 \%$, which is much smaller than the standard deviation of the observed bow shock location.

[14] Figure 5 shows various pressures plotted along the Sun-Mars line for Case $1 . \mathrm{P}_{\mathrm{SW}}$ is the dynamic pressure of the solar wind; $\mathrm{P}_{\mathrm{T}}(\mathrm{sw})$ is the thermal pressure of the solar wind; $\mathrm{P}_{\mathrm{T}}$ (ion) represents the ionosphere thermal pressure; $\mathrm{P}_{\mathrm{B} 0}$ is the magnetic pressure associated with the crustal field and $P_{B}$ is the total magnetic pressure. The small circles indicate the positions of radial grid points. The bow shock can be seen clearly in both the solar wind dynamic pressure and the thermal pressure curves. The transition from a solar wind thermal pressure to magnetic pressure dominated region takes place at around $1.24 \mathrm{R}_{\mathrm{M}}$, which is about the distance that MGS puts the average magnetic pileup boundary (MPB) location at the subsolar line [Vignes et al., 2000]. The total pressure starts to increase significantly until very close to the inner boundary. The pressure gradient is mainly balanced by the magnetic tension force in the region near $\mathrm{X}=1.1 \mathrm{R}_{\mathrm{M}}$. A strong magnetic field permeates the ionosphere; this field is mainly composed of an induced, and mainly y-directed, magnetic field, instead of the crustal magnetic field. The subsolar location of this MPB-like feature is relatively constant and is only effected signifi-

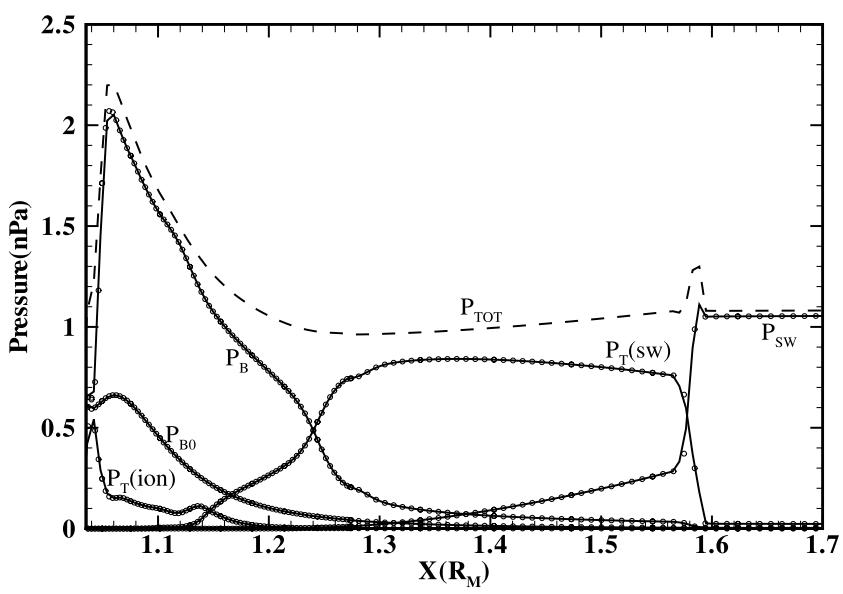

Figure 5. Pressure profiles along Sun-Mars line on the dayside for case $1 . \mathrm{P}_{\mathrm{SW}}$ is the dynamic pressure of the solar wind; $\mathrm{P}_{\mathrm{T}}(\mathrm{sw})$ is the thermal pressure of the solar wind; $\mathrm{P}_{\mathrm{T}}$ (ion) represents the ionosphere thermal pressure; $\mathrm{P}_{\mathrm{B} 0}$ is the magnetic pressure associated with the crustal field and $\mathrm{P}_{\mathrm{B}}$ is the total magnetic pressure. The small circles indicate the positions of radial grid points. 

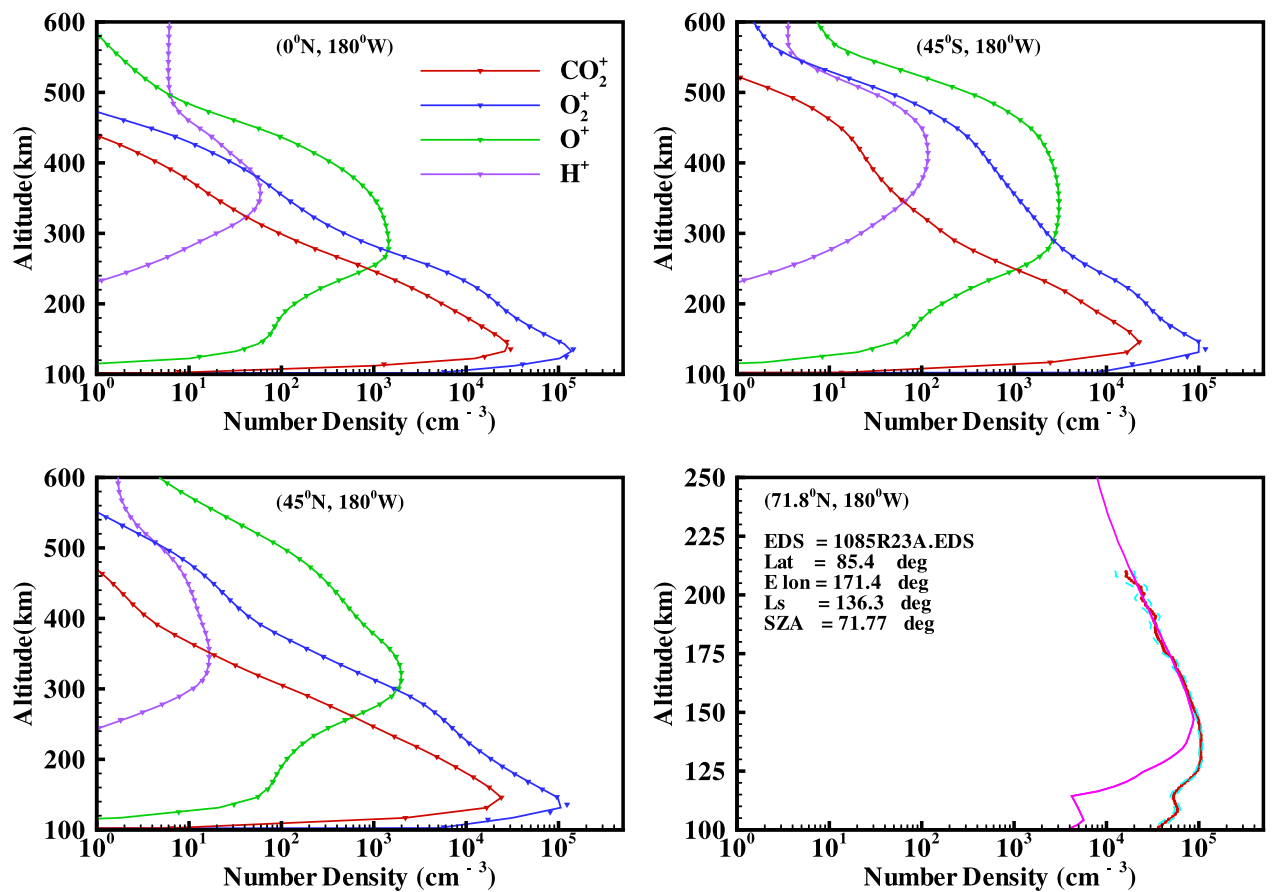

Figure 6. The calculated solar cycle maximum density profiles for case 1 along radial lines for different latitudes in the X-Z plane. The bottom right panel compares an observed Mars Global Surveyor electron density profile (red line) and one-sigma uncertainties (in dashed blue) with the calculated values (green line) at the same solar zenith angle (note the change in altitude scale).

cantly by the local crustal magnetic field, as can be seen from Table 2. A sharp transition from solar wind ion to planetary ion domination takes place at a subsolar distance of $\sim 1.15 \mathrm{R}_{\mathrm{M}}$.

[15] Figure 6 shows the calculated solar cycle maximum (Case 1) ion densities for the subsolar $0^{\circ}, 45^{\circ} \mathrm{N}$, and $45^{\circ} \mathrm{S}$ locations and electron density for $71.8^{\circ} \mathrm{N}$. The ion densities vary at the different locations. The ion densities extended to high altitudes in the presence of strong crustal fields $\left(45^{\circ} \mathrm{S}\right)$. Ion density observations are only available for solar cycle minimum conditions (see Figure 8). However, the MGS radio occultation observations do provide electron density profiles, although only at high latitudes and large solar zenith angles. We selected one representative observation (Lat.: 85.4; SZA: 71.77) and compare it with the calculated values in the bottom right of Figure 6 (note a change in the altitude scale in this figure). The estimated Mars $10.7 \mathrm{~cm}$ flux corresponding to this observation was about 230 (D. Mitchell, private communication, 2004). The agreement between the model results and observation is very good above $\sim 140 \mathrm{~km}$. The disagreement at the low altitudes, we believe, is due mainly to our optical depth approximations, especially the use of a simple cosine factor, at this high zenith angle.

[16] The ideal MHD equations used in the current model are not well suited to calculate the proper plasma temperatures in the ionosphere because the main energy transport mechanism, thermal conduction, is not included. However, these temperatures are important because they have an important role in determining the density distributions. Therefore as mentioned earlier, we added a somewhat "ad hoc" cooling term to arrive at reasonable temperatures, consistent with the observed values. It needs to be noted that the calculated values are pressures and thus the derived temperatures are coupled to the calculated densities. Figure 7 shows these calculated plasma temperatures for three latitudes in the X-Z plane (thus two solar zenith angles) and along the Viking 1 trajectory. These plasma (electron plus ion) temperatures are in the same general range as the observed values (shown by solid squares in the figure) in the relevant altitude range [Hanson et al., 1977; Hanson and Mantas, 1988].

[17] In Figure 8 we plot the solar cycle minimum densities calculated for the Viking 1 conditions (Case 4). Here again we plotted results from the subsolar $0^{\circ}, 45^{\circ} \mathrm{N}$ and

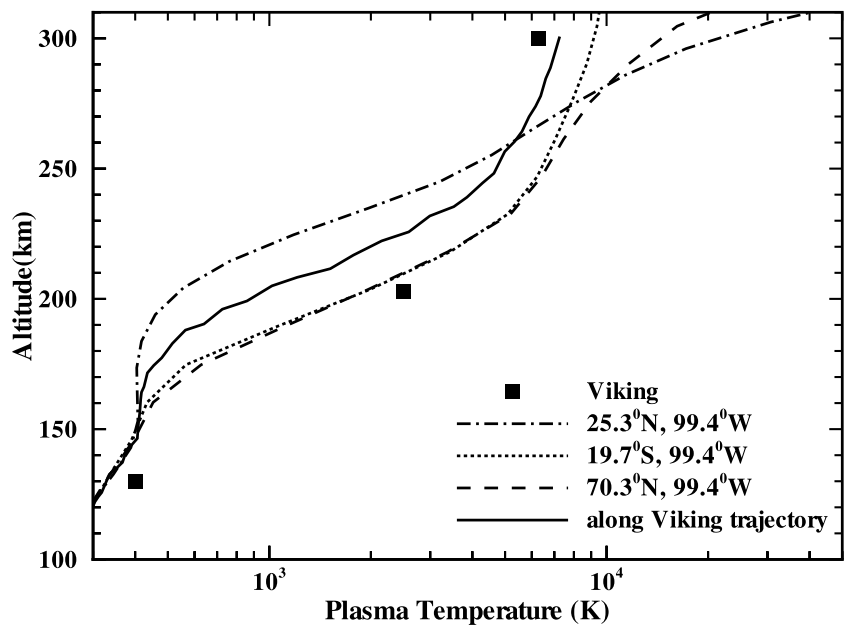

Figure 7. The calculated temperatures along radial lines of constant solar zenith angle (SZA) for case 4. 

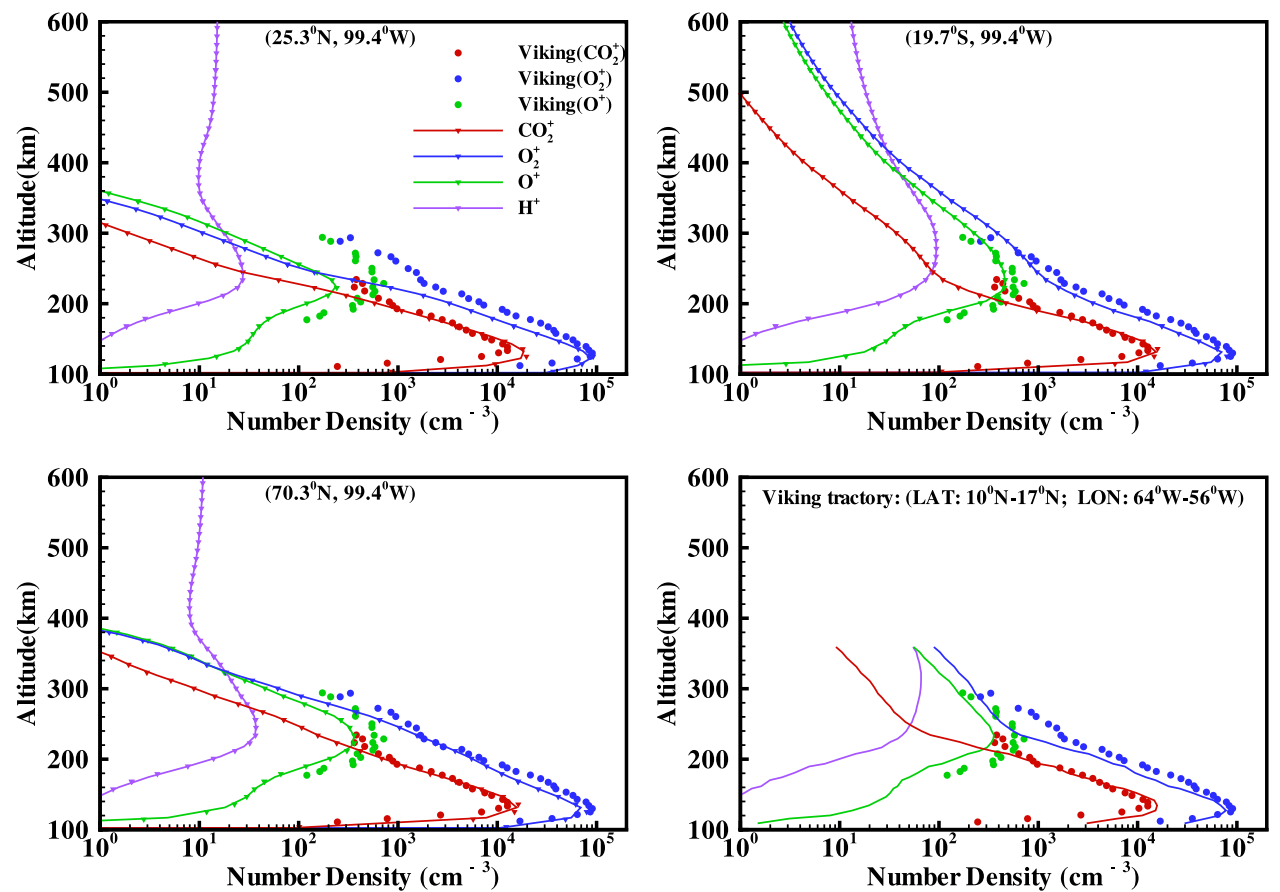

Figure 8. The calculated solar cycle minimum density profiles for case 4 along radial lines for different latitudes in the $\mathrm{X}-\mathrm{Z}$ plane compared with Viking observation.

$45^{\circ} \mathrm{S}$ locations. The bottom right plot shows the results along the Viking 1 trajectory. The measured ion densities from the RPA [Hanson et al., 1977] are also shown in the plots. The agreement between the observed and calculated densities is very good (note that the solar zenith angle corresponding to the Viking 1 measurements was $37-44^{\circ}$ and thus the cosine approximation in the optical depth calculations was a reasonably good one). Here we should also mention that we ran a case for solar cycle minimum conditions with no crustal magnetic field (Case 5). The major difference in the ionospheric results was that above about $250 \mathrm{~km}$ the ion densities are significantly higher in the presence of crustal fields than without such fields.

[18] It has been well established that the transition from chemical equilibrium conditions to transport control takes place near $200 \mathrm{~km}$. In the chemically controlled region above the peak, the plasma density decreases with a scale height, which corresponds to approximately twice that of the major ionizable neutrals [cf. Schunk and Nagy, 2000]. The Viking observations [Nier and McElroy, 1977] estimated the exospheric, solar cycle minimum, neutral gas temperature to be about $185 \mathrm{~K}$, giving $\mathrm{CO}_{2}$ and $\mathrm{O}$ scale heights of about 10.4 and $28.5 \mathrm{~km}$, respectively. The measured Viking $\mathrm{CO}_{2}^{+}$and $\mathrm{O}_{2}^{+}$scale heights were about 23 and $29 \mathrm{~km}$, respectively. Therefore both the observed and calculated densities are consistent with chemical control in this region.

[19] There have been numerous discussions and interpretations about the observed small-scale heights above $200 \mathrm{~km}$ in the region no longer controlled by chemistry. Recently, it has been argued that the presence of horizontal magnetic fields inhibits vertical transport in this region, resulting in the observed scale heights [e.g., Krymskii et al., 2002]. However, Dwivedi and Mahajan [2003] have used Pioneer Venus observations at Venus and suggested that this hori- zontal magnetic field explanation may not hold at Mars. These discussions were based on pure diffusive equilibrium arguments, assuming plasma temperatures constant with altitude. However, significant temperature gradients are known to be present in these regions of the ionosphere and they reduce the "effective" scale height [cf. Schunk and Nagy, 2000]. The one-dimensional, steady state, vertical momentum equation can be written as

$$
\partial \mathrm{p} / \partial \mathrm{r}=-\rho \mathrm{g}-\rho \mathbf{u} \nu .
$$

If we neglect the friction term and use the expression for the scalar pressure $\{p=n k T\}$, equation (8) becomes

$$
\mathrm{kT}(\partial \mathrm{n} / \partial \mathrm{r})+\mathrm{nk}(\partial \mathrm{T} / \partial \mathrm{r})=-\mathrm{nmg} .
$$

Now if the temperature gradient is neglected, the "classical" expression for diffusive equilibrium with the standard expression for the plasma scale height results. However, if the temperature gradient is not neglected, the equation becomes

$$
\frac{1}{\mathrm{n}} \frac{\partial \mathrm{n}}{\partial \mathrm{r}}=-\frac{\mathrm{mg}}{\mathrm{kT}}-\frac{1}{\mathrm{~T}} \frac{\partial \mathrm{T}}{\partial \mathrm{r}} .
$$

Equation (10) clearly indicates that the effect of temperature gradients is to reduce the plasma scale height. We calculated the role of the (dT/dr)/T term, corresponding to the results along the Viking 1 trajectory, shown in Figure 8. We find that this term exceeds the scale height term $[\mathrm{mg} / \mathrm{kT}$ p between $\sim 190$ and $300 \mathrm{~km}$. Furthermore, we calculated the $(\mathrm{dn} / \mathrm{dr}) / \mathrm{n}$ using equation (10) and it matches very closely the calculated values, indicating that, at least at that time, in this limited altitude and latitude region the effects of the crustal field and transport were negligible. Chen et al. [1978] used a one- 


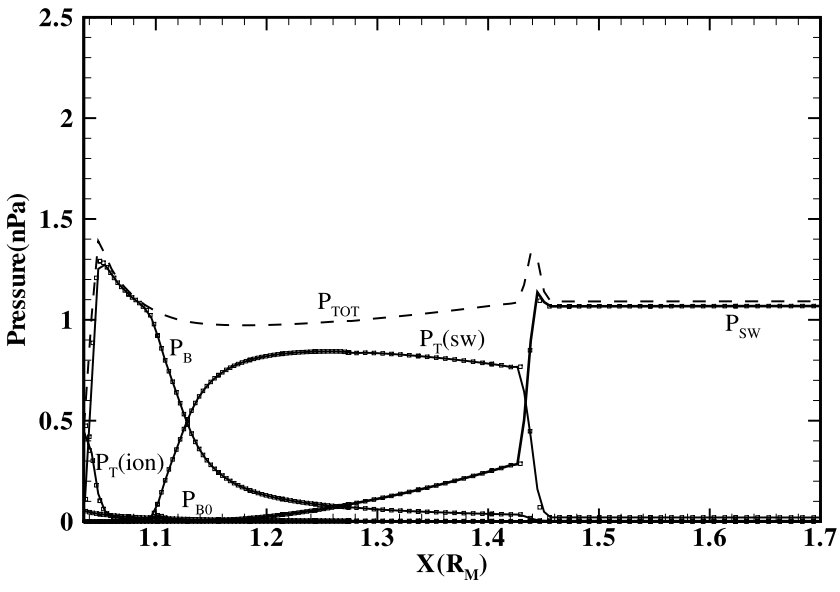

Figure 9. Pressure profiles along Sun-Mars line on the dayside for case 4 (the variables have the same meanings as in Figure 5).

dimensional model which coupled the continuity, momentum and energy equations and assumed no magnetic field. They found that they needed to assume the presence of a strong upward flow in order to reproduce the observed ion density profiles. Shinagawa and Cravens [1989] constructed an MHD model and used observed plasma temperatures. They obtained the best agreement with the observed densities, when they had an induced magnetic field and horizontal removal of plasma (their Case 5). Finally, Fox [1993] constructed a one-dimensional "chemical-diffusive model," in which she also used observed temperatures and concluded that she needed a significant escape flux to have her model results match the observed densities. These past studies, consistent with the current model results, indicate that in general the small, observed scale heights are the result of numerous causes, namely temperature gradients, magnetic fields, and plasma loss due to convective transport.

[20] Figure 9 shows various pressures plotted along the Sun-Mars line for Case 4. The overall features are similar to Figure 5, except that the crustal sources at the subsolar line are much weaker in this case. $\mathrm{P}_{\mathrm{B} 0}$ is close to zero even near the inner boundary. Here again the pressure gradient is mainly balanced by the magnetic tension force in the ionospheric region around $\mathrm{X}=1.1 \mathrm{R}_{\mathrm{M}}$. As can be seen, the bow shock location is much closer to Mars, which is also given in Table 2. Comparison of the bow shock locations between Case 3 and Case 4 shows that the local crustal source is one of the factors that could effect the bow shock locations. The results are also consistent with Figure 1 of Vignes et al. [2002]. The bow shock locations for Case 5 are even closer to the planet, similar to Venus.

[21] We find that the transterminator and escape flux values we obtain with our new model, for both solar cycle maximum and minimum cases, are significantly different from our previously published values, as indicated in Table 3. The main reason for this decrease is that in these current calculations we did a much better job in estimating the temperature dependence of the dissociative recombination rate of $\mathrm{O}_{2}^{+}$, which led to lower ionospheric densities, and which in turn lead to a better agreement with the observed densities. The drop in the calculated densities also led to lower fluxes. The improved altitude resolution also had an effect on the calculated fluxes. The plasma responsible for these fluxes originates mostly in the $250-350 \mathrm{~km}$ region, where transport becomes important and the densities are still relatively high. Inspection of Figure 8 , showing the solar minimum Case 4, clearly shows that $\mathrm{O}_{2}^{+}$is the major ion in this region. This is consistent with the results showing that the $\mathrm{O}_{2}^{+}$flux exceeds the $\mathrm{O}^{+}$one. On the other hand, inspection of Figure 6 , showing the solar maximum Case 1 , shows that $\mathrm{O}^{+}$is the major ion in this region, resulting in mostly $\mathrm{O}^{+}$fluxes. Table 3 also indicates that both IMF orientation and subsolar location have significant effects on the transterminator and escape fluxes. These newly calculated escape fluxes are less than the values estimated from the Phobos measurements, which were obtained around solar cycle maximum conditions [Lundin et al., 1989; Rosenbauer et al., 1989]. However, given the uncertainties associated with the measured values, it seems that this ionospheric source is still likely to make an important contribution to the overall escape flux.

\section{Summary}

[22] We presented the results of model calculations using our four species, spherical MHD model. This new model allowed us to use small $(\sim 10 \mathrm{~km})$ radial grid spacing in the ionospheric region. We found that our calculated ion densities, with parameters corresponding to solar cycle minimum conditions, reproduced the Viking 1 observed ion densities well, as did the solar maximum results compared with the MGS electron density values above about $140 \mathrm{~km}$. The observed plasma scale heights, above $200 \mathrm{~km}$, are likely to be the result of temperature gradients, convective plasma transport, and magnetic field effects. We also found that the calculated bow shock positions for most of the cases considered agree reasonably well with the observed values; the calculated bow shock locations vary with IMF orientation, solar cycle conditions, and subsolar location. The

Table 3. Calculated Transterminator and Escape Fluxes for Solar Cycle Minimum and Maximum Conditions for Nominal Solar Wind Parameters

\begin{tabular}{|c|c|c|}
\hline & Terminator Flux, $\mathrm{s}^{-1}$ & Escape Flux, $\mathrm{s}^{-1}$ \\
\hline $\begin{array}{l}\mathrm{O}_{2}^{+} \\
\mathrm{O}^{+} \\
\mathrm{CO}_{2}^{+} \\
\text {Total }\end{array}$ & $\begin{array}{l}\text { Solar Cycle Maximum (Case 1) } \\
\begin{aligned} 3.4 & \times 10^{23} \\
2.7 & \times 10^{24} \\
4.7 & \times 10^{22} \\
3.0 & \times 10^{24}\end{aligned}\end{array}$ & $\begin{array}{l}2.2 \times 10^{23} \\
1.5 \times 10^{24} \\
3.1 \times 10^{22} \\
1.7 \times 10^{24}\end{array}$ \\
\hline $\begin{array}{l}\mathrm{O}_{2}^{+} \\
\mathrm{O}^{+} \\
\mathrm{CO}_{2}^{+} \\
\text {Total }\end{array}$ & $\begin{array}{l}\text { Solar Cycle Maximum (Case 2) } \\
\begin{aligned} 7.0 & \times 10^{23} \\
4.2 & \times 10^{24} \\
4.7 & \times 10^{22} \\
4.9 & \times 10^{24}\end{aligned}\end{array}$ & $\begin{array}{l}3.2 \times 10^{23} \\
2.5 \times 10^{24} \\
3.7 \times 10^{22} \\
2.8 \times 10^{24}\end{array}$ \\
\hline $\begin{array}{l}\mathrm{O}_{2}^{+} \\
\mathrm{O}^{+} \\
\mathrm{CO}_{2}^{+} \\
\text {Total }\end{array}$ & $\begin{array}{l}\text { Solar Cycle Minimum (Case 3) } \\
\begin{aligned} 6.7 & \times 10^{23} \\
5.9 & \times 10^{23} \\
8.2 & \times 10^{23} \\
1.2 & \times 10^{24}\end{aligned}\end{array}$ & $\begin{array}{l}2.9 \times 10^{23} \\
2.5 \times 10^{23} \\
3.1 \times 10^{22} \\
0.5 \times 10^{24}\end{array}$ \\
\hline $\begin{array}{l}\mathrm{O}_{2}^{+} \\
\mathrm{O}^{+} \\
\mathrm{CO}_{2}^{+} \\
\text {Total } \\
\end{array}$ & $\begin{array}{l}\text { Solar Cycle Minimum (Case 4) } \\
\begin{aligned} 2.5 & \times 10^{24} \\
1.3 & \times 10^{24} \\
2.8 & \times 10^{23} \\
3.8 & \times 10^{24}\end{aligned}\end{array}$ & $\begin{array}{l}1.4 \times 10^{24} \\
8.4 \times 10^{23} \\
1.5 \times 10^{22} \\
2.2 \times 10^{24}\end{array}$ \\
\hline
\end{tabular}


calculated solar cycle minimum and maximum total transterminator and escape fluxes are significantly less than our previously published values. This decrease is mainly due to the change in the ionospheric densities, resulting from a better way of calculating the dissociative recombination rate, which results in lower densities and fluxes. These decreased escape fluxes may still play a significant role in the overall escape from Mars.

[23] Acknowledgments. The authors wish to acknowledge the help of J. L. Fox. The work presented here was supported by NASA grant NAG5-13332.

[24] Arthur Richmond thanks Hiroyuki Shinagawa and another reviewer for their assistance in evaluating this paper.

\section{References}

Acuna, M. H., et al. (1998), Magnetic field and plasma observations at Mars: Initial results of the Mars Global Surveyor Mission, Science, 279, 1676.

Arkani-Hamed, J. (2001), A 50-degree spherical harmonic model of the magnetic field of Mars, J. Geophys. Res., 106, 23,197.

Bauske, R., A. F. Nagy, T. I. Gombosi, D. L. DeZeeuw, K. G. Powell, and J. G. Luhmann (1998), A three-dimensional MHD study of solar wind mass loading processes at Venus: Effects of photoionization, electron impact ionization and charge exchange, J. Geophys. Res., 103, 23,625.

Bougher, S. W., S. Engel, R. G. Roble, and B. Foster (2000), Comparative terrestrial planet thermospheres: 3 . Solar cycle variation of global structure and winds at solstices, J. Geophys. Res., 105, 17,669.

Chen, R. H., T. E. Cravens, and A. F. Nagy (1978), The Martian ionosphere in light of the Viking observations, J. Geophys. Res., 83, 3871.

DeZeeuw, D. L., A. F. Nagy, T. I. Gombosi, K. G. Powell, and J. G. Luhmann (1996), A new axisymmetric MHD model of the interaction of the solar wind with Venus, J. Geophys. Res., 101, 4547.

Dwivedi, A. K., and K. K. Mahajan (2003), Plasma distribution at Venus and ionospheric magnetic fields: Implication for Mars, Geophys. Res Lett., 30(24), 2284, doi:10.1029/2003GL018263.

Fox, J. L. (1993), The production and escape of nitrogen atoms on Mars, J. Geophys. Res., 98, 3297.

Fox, J. L. (2003), Effect of $\mathrm{H}_{2}$ on the Martian ionosphere: Implications for atmospheric evolution, J. Geophys. Res., 108(A6), 1223, doi:10.1029/ 2001JA000203.

Fox J. L., and K. Y. Sung (2001), Solar activity variations of the Venus thermosphere/ionosphere, J. Geophys. Res., 106, 21,305.

Hanson, W. B., and G. P. Mantas (1988), Viking electron temperature measurements: Evidence for a magnetic field in the Martian ionosphere, J. Geophys. Res., 93, 7538.

Hanson, W. B., S. Sanatini, and D. R. Zuccaro (1977), The martian ionosphere as observed by the Viking retarding potential analyzer, J. Geophys. Res., 82, 4351.

Harnett, E. M., and R. M. Winglee (2003), The influence of a minimagnetopause on the magnetic pileup boundary at Mars, Geophys. Res Lett., 30(20), 2074, doi:10.1029/2003GL017852.

Kallio, E., J. G. Luhmann, and J. G. Lyon (1998), Magnetic field near Venus: A comparison between Pioneer Venus Orbiter magnetic field observations and an MHD simulation, J. Geophys. Res., 103, 4753.
Kim, J., A. F. Nagy, J. L. Fox, and T. E. Cravens (1998), Solar cycle variability of hot oxygen atoms at Mars, J. Geophys. Res., 103, 29,339.

Krymskii, A. M., T. K. Breus, N. F. Ness, M. H. Acuna, J. E. P. Connerney, D. H. Crider, D. L. Mitchell, and S. J. Bauer (2002), Structure of the magnetic field fluxes connected with crustal magnetization and topside ionosphere of Mars, J. Geophys. Res., 107(A9), 1245, doi:10.1029/ 2001JA000239

Liu, Y., A. F. Nagy, T. I. Gombosi, D. L. DeZeeuw, and K. G. Powell (1999), 3D multifluid MHD studies of the solar wind interaction with Mars, Geophys. Res., Lett., 26, 2689

Lundin, R., et al. (1989), First measurements of the ionospheric plasma escape from Mars, Nature, 341, 609

Ma, Y., A. F. Nagy, K. C. Hansen, D. L. DeZeeuw, and T. I. Gombosi (2002), Three-dimensional multispecies MHD studies of the solar wind interaction with Mars in the presence of crustal fields, J. Geophys. Res., 107(A10), 1282, doi:10.1029/2002JA009293.

Nagy, A. F., et al. (2004), The plasma environment of Mars, Space Sci. Rev., 111(1-2), 33

Nier, A. O., and M. B. McElroy (1977), Composition and structure of Mars' upper atmosphere: Results from the neutral mass spectrometer on Viking 1 and 2, J. Geophys. Res., 82, 4341.

Powell, K. G., P. L. Roe, T. J. Linde, T. I. Gombosi, and D. L. DeZeeuw (1999), A solution-adaptive upwind scheme for ideal magnetohydrodynamics, J. Comp. Phys., 154, 284.

Rosenbauer, H., et al. (1989), Ions of Martian origin and plasma sheet in the Martian magnetosphere: Initial results of the TAUS experiment, Nature, 341,612 .

Sauer, K., A. Bogdanov, and K. Baumgartel (1994), Evidence of an ion composition boundary (protonopause) in bi-ion fluid simulations of solar wind mass loading, Geophys. Res. Lett., 21, 2255.

Sauer, K., E. Dubinin, and K. Baumgartel (1998), Nonlinear MHD waves and discontinuities in the Martian magnetosheath: Observations and 2D bi-ion MHD simulations, Earth Planets Space, 50, 793.

Schunk, R. W., and A. F. Nagy (2000), Ionospheres, Cambridge Univ. Press, New York.

Shinagawa, H., and T. E. Cravens (1989), A one-dimensional multispecies magnetohydrodynamic model of the dayside ionosphere of Mars, J. Geophys. Res., 94, 6506.

Steinolfson, R. S., and S. Cable (1993), Venus bow shocks at unusually large distances from the planet, Geophys. Res. Lett., 20, 755.

Tanaka, T., and K. Murawski (1997), Three-dimensional MHD simulation of the solar wind interaction with the ionosphere of Venus: Results of two-component reacting plasma simulation, J. Geophys. Res., 102, 19,805 .

Vignes, D., et al. (2000), The solar wind interaction with Mars: Location and shapes of the bow shock and magnetic pileup boundary from observations of the MAG/ER experiment onboard Mars Global Surveyor, Geophys. Res. Lett., 27, 49.

Vignes, D., et al. (2002), Factors controlling the location of the bow shock at Mars, Geophys. Res. Lett., 29(9), 1328, doi:10.1029/2001GL014513.

K. C. Hansen, Y. Ma, A. F. Nagy, and I. V. Sokolov, Space Physics Research Laboratory, Department of Atmospheric, Oceanic, and Space Sciences, University of Michigan, Ann Arbor, MI 48109, USA. (kenhana umich.edu; yingjuan@umich.edu; anagy@umich.edu; igorsok@umich. edu) 